\title{
Design of a dry inorganic substances dispenser
}

\author{
Dmitry A. Filippov ${ }^{1, *}$, and Arkadiy V. Moiseev ${ }^{1}$ \\ ${ }^{1}$ FSBEI HE Kuban State Agrarian University named after I.T.Trubilin, Krasnodar, Russian Federation
}

\begin{abstract}
The article is devoted to the introduction of dry inorganic substances, mixtures, complex solids on field surface or on the object of treatment by a universal dispenser. The article has a research character which is expressed in the fact that the analysis of methods and means for the introduction of dry inorganic substances as well as extracts from the works of leading scientists on the subject are given, promising scientific developments in the form of patent analysis which provide optimal flow of the technological process are shown, their shortcomings and solutions to these problems are pointed. There was justified the main tendency of development of dispensers and improvement of their designs for advancing the quality rates of their work. Also, there was shown the type of the proposed design, its description and the flow of the technological process. As a result of the work done, there was obtained the design of the dispenser of dry inorganic substances with proposed working elements. The conclusions set out the main results achieved so far.
\end{abstract}

Kuban State Agrarian University has always occupied one of the leading places in the development of devices in the field of agricultural mechanization. The faculty of agricultural mechanization and, in particular, the department of "Processes and machines in agribusiness" takes one of the leading roles in this process. The department is actively working on the creation of agricultural machines for mechanization of small farms. It is necessary to adhere to all agrotechnical requirements for the cultivation of a certain culture in the process of growing crops.

The use of fertilizers is one of the main conditions for the development of agriculture. At present time, the volumes of application of fertilizers has not yet reached the global level in agriculture of our country and a sound science-based approach to fertilization is required for providing the necessary gross yield of agricultural products. Under these conditions the efficiency of the use of mineral fertilizers and mobilization of soil fertility to maintain crop yields at a sufficiently high and desirable level can be achieved only taking into account all soil, weather and agricultural factors in adherence to the technological processes of storage, preparation and application of fertilizers.

Over the past few years, despite the alleged increase in crop production, many rates in the country are below the agricultural production market average. At the same time, it should be taken into account when crops are obtained only at the expense of natural fertility

\footnotetext{
* Corresponding author: sergey belousov87@mail.ru
} 
and small amounts of fertilizers, a negative balance of nutrients is created in soil, and this ultimately leads to the inevitable depletion of the latter [1].

Traditional technologies of cultivation of agricultural crops imply the introduction of averaged doses of fertilizers in the system of crop rotations, without taking into account the variability of soil fertility and state of crops, but this approach no longer meets the requireme9nts of today.

Agrotechnical operations on application of mineral fertilizers are an important part in almost any agricultural technology. In addition, this operation, as a rule, is a significant part of the cost of entire technological process of cultivation of agricultural crops and as a consequence - the cost of a final product. Also, the application of mineral fertilizers significantly affects the environmental situation in the field, which in turn affects the soil fertility. It is obvious that the correct calculation of the fertilizer dose and the exact place of its application are the most important tasks in the production of crop products [2].

To apply fertilizers to the field surface, hinged, trailed and self-propelled machines are used as well as rod sowing machines equipped with centrifugal, pneumatic or screw dispensing systems.

Centrifugal disks with vertical and horizontal axes of rotation, pendulum, pneumatic and centrifugal-pneumatic devices as well as belt spreaders are used as working elements of the dispersal-type devices. Devices of the dispersal type give kinetic energy to particles dispersing them and then spread out them in the form of a fan or a jet. The particles gain the initial velocity, the vector of which is generally directed at an angle to a horizon. Aggregates of this type have a relatively high efficiency but the rates of uniformity of dispension require their improvement.

Modern manufacturers of agricultural machinery produce their products oriented mainly to a "large" producer. In this regard, the means of small mechanization are now more relevant than ever. This confirms the increased demand for motor cultivators and nomenclature of choice of hinged equipment to them. They are attractive for its affordability in pricing policy, because the starting price is from a few thousand rubles. However, not all entry-level farmers especially in private farms understand how to move from traditional tractors to this simple but very profitable investment as a motor cultivator. It is so versatile that it can perform almost all the work associated with the production of agricultural products ranging from soil tillage to dispersal of forage on farms. It can be used on a small area of up to 3 hectares or in a limited space (garden in a private farm or fenced cultivation) [4].

As a result of the market analysis there was revealed the basic concept of the trend of equipment development for motor cultivators as the main power means of small mechanization. The range of equipment offered by modern inventors is quite wide and is based on the following principles (Figure 1). 


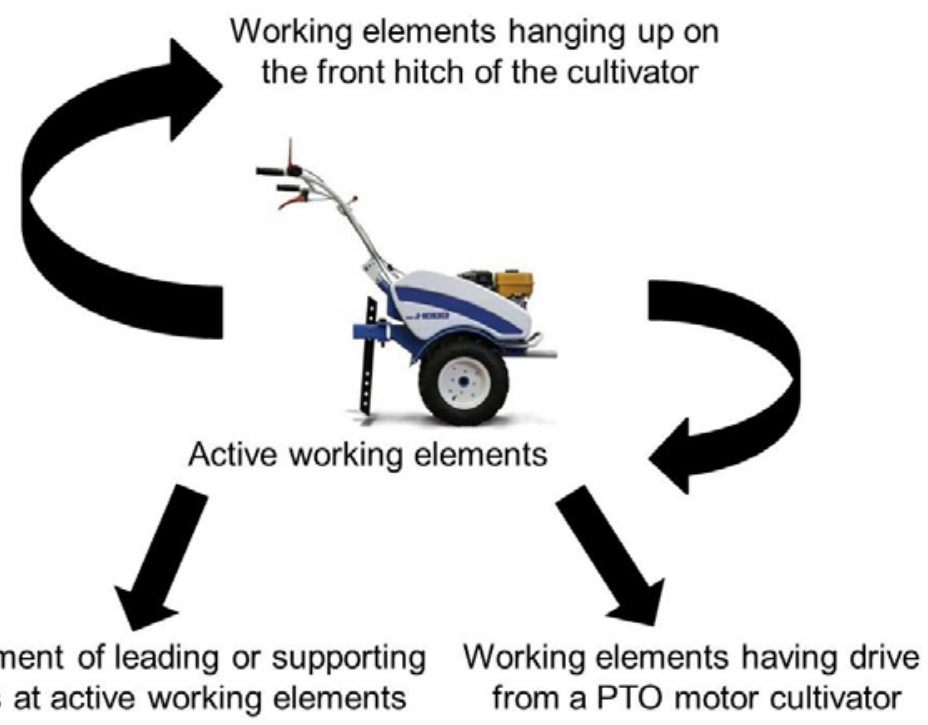

Fig. 1. Scheme of motor-cultivator equipment application.

In the a result of the review of literary sources, the leading scientists recommend using the change of working elements on the motor cultivator, for example, as a replacement of support running wheels for cutters and soil treatment, although there is the equipment that works from the PTO motor cultivator. These include almost all motor cultivators designed for their use in private farms. Their weight usually ranges from 50 to $100 \mathrm{~kg}$ and the engine capacity ranges from 3.5 to $8 \mathrm{hp}$. The reduction gear typically has two forward and one rear speeds. Sometimes they also can be divided into two by changing the diameter of the drive pulley. Thus, there are four front and two rear speeds. Motor cultivators of light and middle classes are rarely equipped with PTO, the belt drive pulley on the motor shaft or reduction gear are more often used for this purpose [5].

Based on the data of long-term experiments when growing a particular crop, farmers have developed an average value for the application of different types of fertilizers and the use of various technological operations. After all, the quantity and quality of the harvest depends on adherence to all operations for the crop cultivation.

The fertilizers offered for sale according their aggregate state are divided into: powdered, granulated, macrocrystalline, liquid, suspended. Granulated and macrocrystalline fertilizer has several advantages compared to powdered, easier to store, easier to introduce into soil with fertilizer sowing machines and cultivators, but the most important is that it gives high yields in most soils [6].

The above mentioned methods and methods of application of mineral fertilizers are designed for use on large areas and spaces. However, the use of these technologies is impossible in conditions of limited land use, the existing machines and units cannot produce the accurate dosed introduction of the preparation exactly in the right place and do not have the possibility of the uniform introduction of the preparation over the entire width of the grip [7].

The use of a small-sized dispenser of dry inorganic mixtures in conditions of limited land use will significantly improve the quality of the products and increase the productivity of this technological operation. Technically, the result is achieved in when the dispenser is mounted on the front portion of the motor cultivator and consists of a hopper, unloading 
window with a dispenser with a reflective screen, has the drive of the working elements as from the PTO shaft of the cultivator and as the support-driving wheels by means of belt or chain transmission, the hopper includes the unloading window with the possibility of dosed introduction of the preparation, the centrifugal disk has a fertilizer dispenser with a changing top angle and a fertilizer dispenser for the introduction of preparation into soil and the rear hitch of the motor cultivator contains a support wheel and an adjustable counterweight plate [8].

In practice, the present design will expand the range of applications of motor cultivators and the design feature of the dispenser provides the increase of efficiency of the introduction of dry inorganic mixtures in conditions of limited land use, which will significantly improve the quality of the products and increase the productivity of this technological operation [9].

The dispenser works as follows (Figure 2): in the process of the unit's motion in the field the dispenser of mineral fertilizers mounted on the front hitch 3 of the motor cultivator with a reflective screen 11 bedded on the supporting-driving wheels 1 , seed material from the hopper 9 is naturally poured through the hopper 8 on the centrifugal disk 5 , seed material with the help of fertilizer dispenser 7 with a variable top angle is supplied to the blades 6 located on the centrifugal disk 5 and deposited on the surface of the field. The centrifugal disk 5 is driven through an angular reduction gear 4 by means of a chain or belt drive 2, from support-drive wheels 1 or from the PTO of the motor cultivator 10 . On the rear hitch of the motor cultivator 12 was mounted a counterweight 13 with a support wheel 14 which can implement the adjustment depending on the weight of seeds in the hopper. The hopper is made in a plastic frame with a metal base to reduce its weight and steel intensity [10].

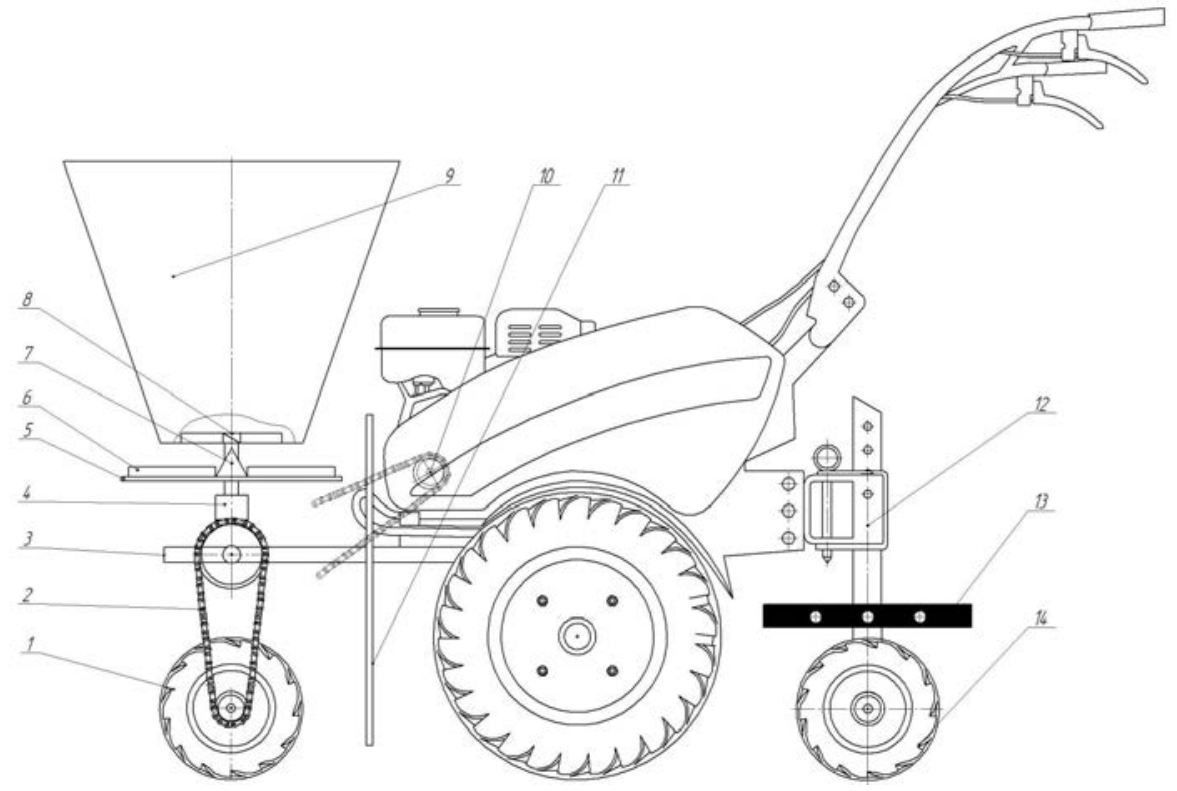

Fig. 2. Schematic diagram of dry inorganic substances dispenser design.

As a result, it is possible to indicate the direction of a further work. It will be connected with the improvement of the process of applying mineral fertilizers and dry inorganic mixtures in the conditions of limited space, manufacture and design of working elements to the motor cultivators are urgent, especially when working with such working elements in limited space. Many agricultural machinery manufacturers are focused on a large 
manufacturer, and production of working elements to the motor cultivator become a subject of farmers' concern.

\section{References}

1. Trubilin E.I. Results of experimental studies determining the degree of traction resistance of the plowshoe in the treatment of heavy soils / Trubilin E.I., Belousov S.V., Lepshina A.I. // The Political Network Electronic Scientific Journal of the Kuban State Agrarian University. -2014. -No. 103.-C. 673-686.

2. Maslov, G. G., Pleshakov, V. N. Prediction of the technical level of domestic and foreign technology [Text] / Engineering in agriculture. - 2001. - № 5. - p. 31-32.

3. Kulintsev, V.V. The fundamentals of the new generation agriculture system in the Stavropol Territory: monograph / V.V. Kulintsev, E.I. Godunova, L.I. Zhelnakova. Stavropol: AGRUS Stavropol GAU. - 2013. - 96 p.

4. Rykov, V.B. Development of efficient mobile technological unitsfor the conditions of southern Russia / V.B. Rykov, S.I. Kambulov, E.I. Trubilin, N.V. Shevchenko Krasnodar, 2019. - 265 s.

5. Parkhomenko G.G. Perfection of working organs for tillage / Parkhomenko GG, Bozhko I.V., Semenikhina Y.A., Pantyukhov I.V., Drozdov S.V., Gromakov A.V., Kambulov S.I., Belousov S.V. // State and prospects of the development of agricultural machinery. Collection of articles of the 9th international scientific and practical conference within the framework of the 19th international agro-industrial exhibition "Interagro-mash-2016". -2016. -FROM. 27-30.

6. Pakhomov, V.I. Experience in the cultivation of winter wheat in conditions of insufficient moisture / V.I. Pakhomov, V.B. Rykov, S.I. Kambulov, N.V. Shevchenko, E.L. Revyakin. - Moscow, 2015. - 160 p.

7. Belousov S.V. Patent search for structures that provide soil treatment with the turnover of the reservoir. Search method. The proposed technical solution / Belousov S.V. // The political network electronic scientific journal of the Kuban State Agrarian University. -2015. No. 109.-C. 416-450.

8. Rykov, V.B. Features of the cultivation of cereals in conditions of insufficient moisture / V.B. Rykov, S.I. Kambulov, G.G. Parkhomenko / Sat. "Ecology and agricultural technologies: agroengineering solutions" materials of the 7th International Scientific and Practical Conference. - 2011. - p. 86-92.

9. Pakhomov, V.I. Technologies of applying organic, mineral, microelement fertilizers to increase the yield and quality of crop production under conditions of insufficient moisture / Pakhomov V.I., Rykov V.B., Kambulov S.I., Vyalkov V.I., Shevchenko N.V., Ksenz A.Y., Mazhara V.M. - Zernograd, State Scientific Institution SKNIIMESH Russian Agricultural Academy. - 2013. - 140 p. 